\title{
T-Cadherin Expression in Actinic Keratosis Transforming to Invasive Squamous Cell Carcinoma
}

\author{
Stanislaw A. Buechner ${ }^{a} \quad$ Therese J. Resink $^{b}$ \\ ${ }^{a}$ Histologische Diagnostic GmbH, Basel, Switzerland; ${ }^{b}$ Department of Biomedicine, \\ Basel University Hospital, Basel, Switzerland
}

\section{Keywords}

Actinic keratosis · Invasive squamous cell carcinoma $\cdot$ Transformation · T-cadherin

\begin{abstract}
Background/Aims: Clinical and histological features of actinic keratosis (AK) cannot predict malignant transformation to invasive squamous cell carcinoma (iSCC) in individual lesions. We investigated whether patterns/distribution of T-cadherin in AK lesions have biomarker value in predicting transformation to iSCC. Methods: 28 specimens of cutaneous iSCC exhibiting adjacent or overlying AK were immunostained for T-cadherin and classified according to AK histological grade (AK I-III) and basal growth pattern (PRO I-III). Results: T-cadherin staining was absent/very weak in 16 and strongly positive in 12 cases. iSSCs lacking T-cadherin expression were most commonly (12/16 cases) associated with type AK I or PRO I lesions, whereas the majority (10/12 cases) of T-cadherin-positive iSCCs originated from AK II and AK III/PRO II and PRO III. In T-cadherin-negative iSCCS, T-cadherin expression was absent in overlying AK and early invasive tumour but retained in AK areas adjacent to the tumour. In contrast, T-cadherinpositive iSCCs displayed expression of T-cadherin in the adjacent AK and early invasive tumour. Conclusions: T-cadherin-negative iSCC arises from AK showing partial or extensive regional loss of T-cadherin in the basal layer of the epidermis. We speculate that T-cadherin loss in individual AK lesions could indicate potential transformation of AK into aggressive iSCC.
\end{abstract}

(C) 2019 The Author(s)

Published by S. Karger AG, Basel

\section{Introduction}

Actinic keratoses (AKs) are intraepithelial atypical proliferations of keratinocytes representing the most common potentially serious manifestations of long-term sun exposure [1, 2]. Although AK is generally considered a low-risk disease, AKs are a strong risk factor for the development of squamous cell carcinoma (SCC), both in the general population and in immu- 
nosuppressed transplant recipients [3-5]. Because AK and SCC have a similar genetic profile and AKs at the level of cytology are frequently indistinguishable from SCC in situ, AK is viewed as being synonymous with superficial SCC in situ $[6,7]$. An established histological classification system devised by Röwert-Huber et al. [6] divides AK into three categories based on extent of atypical keratinocytes throughout the epidermis: early in situ SCC type AK I where atypical keratinocytes are found in the basal and suprabasal layer of the epidermis, early in situ SCC type AK II with crowded atypical keratinocytes extending to the lower two thirds of the epidermis and in situ SCC type AK III exhibiting atypical keratinocytes that extend to more than two thirds of the entire thickness of the epidermis with a complete loss of maturation. However, the extent of upward epidermal dysplasia is no predictor of AK dysplastic severity or malignant potential: all AK lesions, irrespective of intraepidermal neoplasia thickness, may potentially undergo malignant transformation into invasive SCC (iSCC) [8].

Very recently, and given the evidence for a predominant association of type AK I with iSCC [2, 8-11], Schmitz et al. [12] suggested that downward-directed proliferation of atypical basal cells may be independent of the extent of atypical keratinocytes throughout different layers of the epidermis. They proposed a complementary classification system based on three distinct downward-directed basal epidermal growth patterns of AK: crowding of basal atypical keratinocytes (PRO I), slight budding of atypical keratinocytes into the upper papillary dermis and formation of round nests of atypical keratinocytes (PRO II), or spiky or filiform papillary elongation of atypical keratinocytes protruding into the upper dermis and exceeding the thickness of the overlying epidermis (PRO III, papillary sprouting). Using this morphological system, AKs presenting with advanced basal growth patterns (budding/PRO II and sprouting/PRO III) were most commonly found adjacent to iSCCs [9]. To assist risk stratification, Schmitz et al. [12] proposed an AK classification adjustment that considers both upwards and downwards growth patterns. However, the predictive value of a combinatorial application of the two classification systems has yet to be tested in clinical practice, and on the basis of clinical and histopathological features it is currently not possible to predict if, or when, any given AK lesion will progress into iSCC.

We believe that prediction of potential malignant transformation in individual AK lesions could be assisted by the establishment of biomarker signatures enabling early recognition of potential malignant cellular behaviour. Cadherins represent a superfamily of transmembrane proteins that mediate calcium-dependent intercellular adhesion and have essential functions in tissue morphogenesis and homoeostasis [13]. The roles of the classical and desmosomal cadherins have been extensively studied in relation to the regulation of keratinocyte behaviour in the progression of benign and malignant skin diseases $[14,15]$. A cadherin superfamily member less well studied in the context of the skin is T-cadherin, which uniquely lacks transmembrane and cytoplasmic domains and is anchored to the cell membrane via a glycosylphosphatidylinositol moiety [16]. T-cadherin is strongly expressed on epidermal basal layer keratinocytes of human [17-19] and murine skin [17]. As the keratinocytes move away from the basement membrane, they lose their capacity for cell division and stop expressing T-cadherin [17-19]. T-cadherin is commonly expressed in AK, albeit with marked regional loss in some specimens [20,21], and it is prominently upregulated in basal cell carcinoma, a hyperproliferative disorder of basaloid cells [18]. In contrast, in Bowen disease, considered a high-risk and potentially malignant in situ SCC [22], expression of T-cadherin on the basal cell layer is broadly weakened or absent compared with the adjacent normal epidermis [20], while in SCC downregulation of T-cadherin associates with histological features of malignant transformation from non-invasive to iSCC [20]. In this study we investigated associations between absence or presence of T-cadherin expression in iSCC and AK basal growth pattern. We also assessed whether the pattern and distribution of T-cadherin in AK lesions might have prognostic value in assessing potential AK transformation to iSCC. 

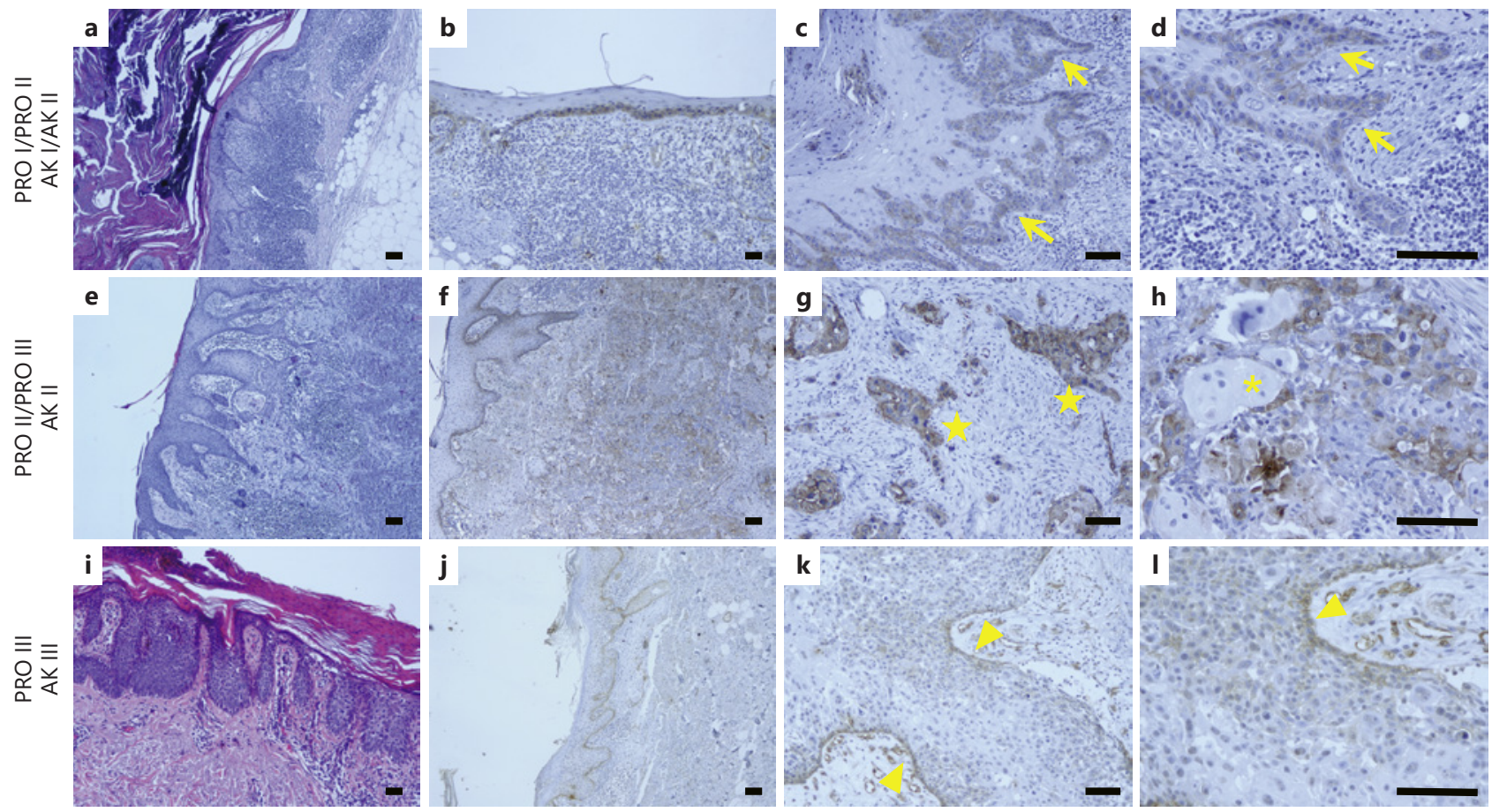

Fig. 1. Haematoxylin and eosin staining (a, e and i) and immunostaining for T-cadherin ( $\mathbf{b}-\mathbf{d}, \mathbf{f}-\mathbf{h}$ and $\mathbf{j}-\mathbf{I})$ in $\mathrm{T}$-cadherin-positive invasive squamous cell carcinoma (iSCC) specimens (scale bars $=100 \mu \mathrm{m}$ ). The majority (10 of 12) of cases exhibited AK II/AK III grading together with PRO II (e-h) or PRO III (i-I) growth patterns. In all cases T-cadherin was commonly expressed in the basal layer keratinocytes of AKs (b, $\mathbf{f}$ and $\mathbf{j})$. Within iSCCs T-cadherin was present in dermal tumour nodules (stars, $\mathbf{g}$ ), the peripheral layer of nodules (arrowheads, $\mathbf{k}$ and $\mathbf{I}$ ) and the invasive front of tumours (arrows, $\mathbf{c}$ and $\mathbf{d}$ ). Tumour cells in the lesion centre were occasionally negative (asterisk, $\mathbf{h}$ ).

\section{Materials and Methods}

Skin specimens were retrieved from archives of the private laboratory Büchner Histologische Diagnostik GmbH, Basel, Switzerland. Only cases with recognizable AK adjacent to, or overlying, the iSCC were selected for this study: specimens (including both biopsies and excisions) from a total of 28 patients were available. Ten normal skin specimens obtained from the samples of wide surgical resection of cutaneous tumours served as controls. Routine lightmicroscopic and immunohistochemical studies were performed on formalin-fixed, paraffinembedded skin specimens. Immunohistochemical stains were performed on 4 - $\mu$ m-thick sections using a universal immunoperoxidase polymer detection system (N-Histofine Simple Stain MAX PO; Nichirei Biosciences, Tokyo, Japan). Sections were deparaffinized in xylol and hydrated through grades of ethanol, and then heated in a citric acid buffer $(\mathrm{pH}, 6.0)$ at $95^{\circ} \mathrm{C}$ for $60 \mathrm{~min}$ for antigen retrieval. Endogenous peroxidase was blocked with $0.3 \%$ hydrogen peroxide for $10 \mathrm{~min}$. The primary antibodies used were goat anti-T-cadherin (1:600; cat. \# AF3264; R\&D Systems Europe Ltd., Abingdon, UK). Sections were incubated with the primary antibodies at room temperature for $60 \mathrm{~min}$. Immunoreactivity was visualized by using the Histofine Simple Stain MAX PO reagent and diaminobenzidine tetrahydrochloride as the substrate. Sections were counterstained with Mayer's haematoxylin, dehydrated with grades of ethanol, cleared with xylene, and mounted with Tissue-Tec coverslipping film. For negative controls, the primary antibody was omitted from the staining protocol. All immunohisto- 

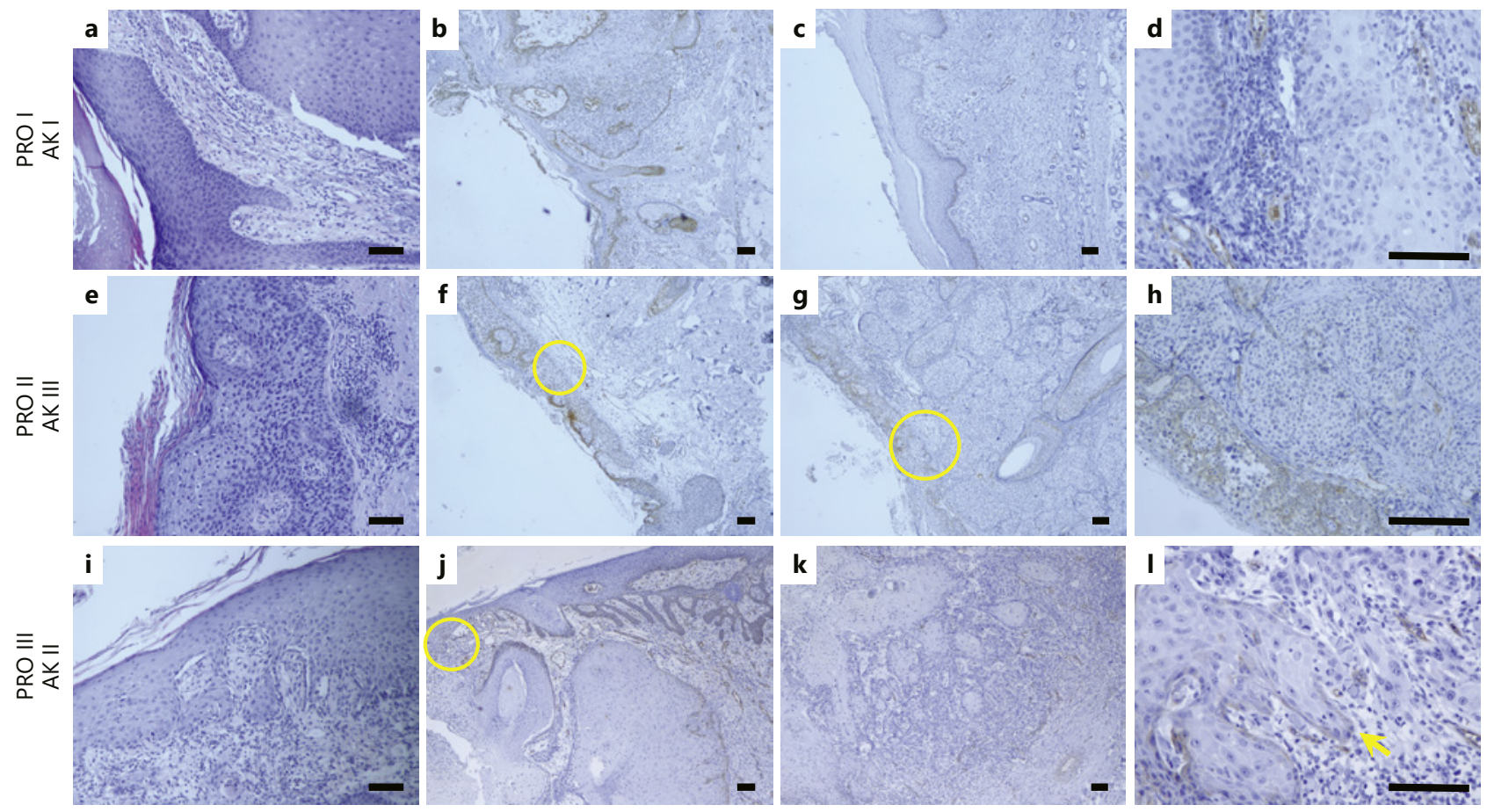

Fig. 2. Haematoxylin-eosin staining (a, e and $\mathbf{i})$ and immunostaining for $\mathrm{T}$-cadherin $(\mathbf{b}-\mathbf{d}, \mathbf{f}-\mathbf{h}$ and $\mathbf{j}-\mathbf{I})$ in $\mathrm{T}$ cadherin-negative iSCC specimens (scale bars $=100 \mu \mathrm{m}$ ). The majority of cases (12 of 16$)$ exhibited AK I grading and PRO I basal growth patterns (a). Although T-cadherin was present within the basal layer of AKs (b, c, $\mathbf{f}-\mathbf{h}$ and $\mathbf{j}$ ), frequent zonal loss or weakening of T-cadherin expression was observed (circles, $\mathbf{f}, \mathbf{g}$ and $\mathbf{j}$ ). Within iSCCs T-cadherin expression was completely absent or only very weakly diffuse in a few atypical keratinocytes at the invasive front of tumours (arrow, I).

chemical reactions were run in duplicate. For each sample, a representative section of the skin was photographed for image analysis. The study was approved by the regional ethics committee (Ethikkommission beider Basel, EKBB 287/09).

\section{Results}

All 28 cases of cutaneous iSCCs examined originated from basal proliferative AK histologically classified as AK I-III. 12 iSCCs were strongly positive for T-cadherin, whereas in 16 iSCCs T-cadherin expression was either very weak or absent. Of the 12 cases of T-cadherinpositive iSCCs, 2 were associated with both a crowding of atypical keratinocytes limited to the basal layer of the epidermis as well as downward budding of dysplastic keratinocytes (PRO I and PRO II) (Fig. 1a-d). These cases were histologically characterized as type AK I and/ or AK II. 10 of the T-cadherin-positive cases were associated with either downward dysplasia exhibiting protruding nests of atypical keratinocytes (PRO II) or filiform papillary elongation of atypical keratinocytes (PRO III) (Fig. 1 e-l). These cases were histologically classified as type AK II or AK III. Of the 16 cases of T-cadherin-negative iSCCs, 12 exhibited a crowded basal dysplasia (PRO I) (Fig. 2a-d). These cases were histologically characterized as type AK I. The remaining 4 cases of T-cadherin-negative iSCCs exhibited downward directed budding (PRO II; Fig. 2e-h) and/or sprouting basal dysplasia (PRO III; Fig. 2i-l). These cases were histologically classified as type AK II or AK III. 

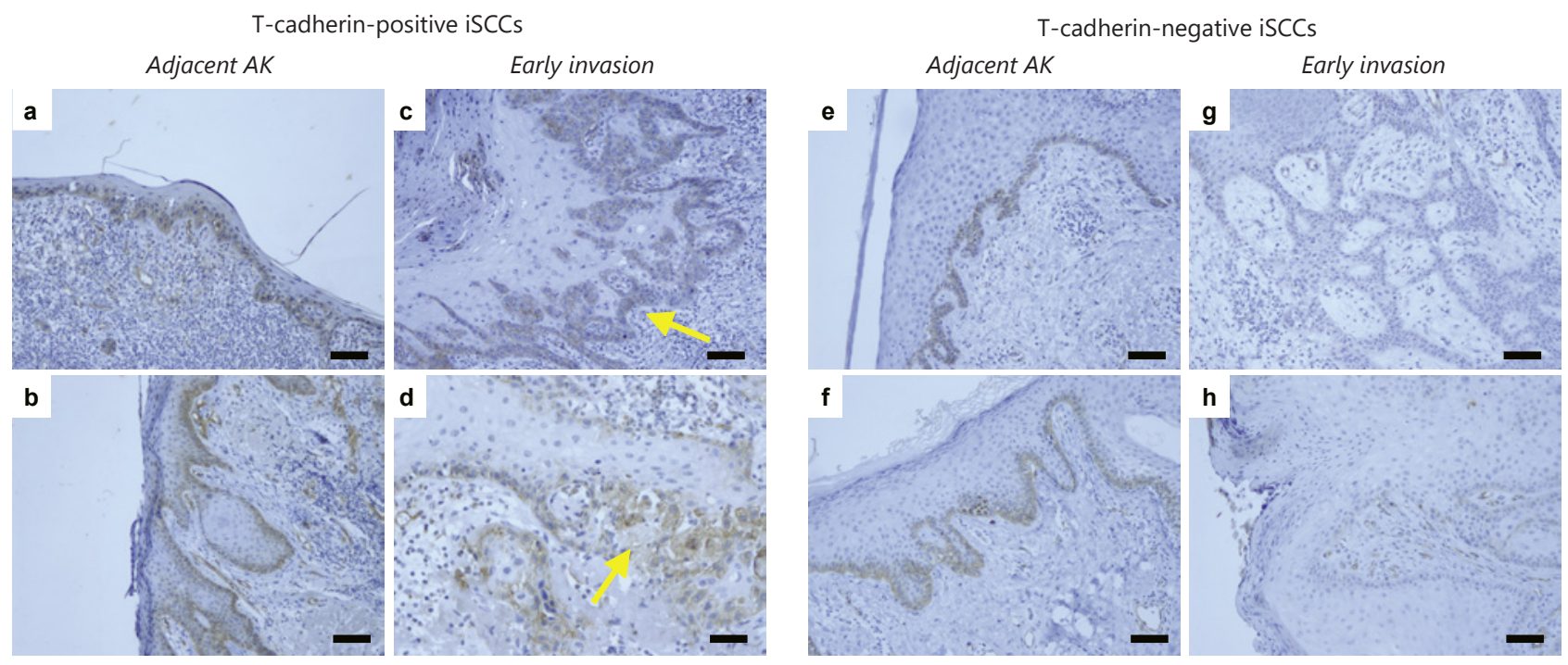

Fig. 3. Patterns and distribution of $\mathrm{T}$-cadherin expression in adjacent $\mathrm{AK}$ and early invasive $\mathrm{AK}$ (scale bars = $100 \mu \mathrm{m})$. T-cadherin was strongly expressed in adjacent AK of both T-cadherin-positive (a, b) and T-cadherin-negative iSCCs $(\mathbf{e}, \mathbf{f})$. In T-cadherin-positive iSCCs, T-cadherin was present in peripheral invasive fronts of tumours irrespective of downward AK growth patterns $(\mathbf{a}, \mathbf{b})$ or occurrence of early invasion (arrows, c, d). In T-cadherin-negative iSCCs, T-cadherin expression was absent in overlying AK exhibiting early invasive characteristics $(\mathbf{g}, \mathbf{h})$.

In T-cadherin-positive iSCCs the adjacent AK retained T-cadherin expression in basal dysplastic keratinocytes irrespective of the downward AK growth patterns (Fig. 3a, b) or occurrence of early invasion (Fig. 3c, d). In contrast, for T-cadherin-negative iSCCs a loss of T-cadherin expression was observed in areas of AK exhibiting early invasive characteristics (Fig. 3g, h) although the AK areas adjacent to the tumour retained T-cadherin expression (Fig. 3e, f).

When present within iSCCs, T-cadherin expression was usually strong in the invasive superficial front of the tumours (arrows in Fig. 1c, d, Fig. 3c, d). Dermal tumour nodules showed either diffuse strong T-cadherin positivity (stars in Fig. 1g) or were predominantly positive in the peripheral layer of the nodules (arrowheads in Fig. 1k, l) while being negative for T-cadherin in some tumour cells in the centre of the lesions (asterisk in Fig. 1h).

\section{Discussion}

This study has found that iSSCs lacking T-cadherin expression are most commonly associated with early in situ type AK I or PRO I lesions, whereas the majority of T-cadherin-positive iSCCs originated from AK II and AK III/PRO II and PRO III. Additionally, AKs adjacent to T-cadherin-negative iSCCs predominantly (12 of 16) exhibited PRO I characteristics, while AKs adjacent to T-cadherin-positive iSCCs predominantly (10 of 12 cases) exhibited PRO II and III characteristics. Together these observations indicate that the greater grades of AK downward expansion and upward dysplasia associate with T-cadherin-positive iSCCs. In contrast, AK dysplasia in T-cadherin-negative iSCCs was limited to the basal layer. Therefore, even in early in situ AKs that lack significant downward proliferation a loss of T-cadherin may be a potential indicator of an iSCC phenotype. On the other hand, the remarkable preservation of T-cadherin in AKs showing pronounced downward proliferation and upward dysplasia of atypical keratinocytes suggests that iSCCs originating from T-cadherin-positive AKs may 
show a less aggressive course of progression than those iSCCs deriving from T-cadherinnegative AK lesions.

Although this study has not been able to demonstrate that iSCCs deriving from T-cadherinnegative AKs follow a more malignant course, previous in vivo and in vitro investigations have shown that T-cadherin negativity in cutaneous SCC is associated with more malignant behaviour $[20,23,24]$. We showed that regional loss of T-cadherin expression was more prominent and frequent in SCC classified as moderately to poorly differentiated than in SCC classified as well differentiated [20]. However, in both categories aberrant and/or absence of T-cadherin expression was associated with histological features of a potentially more malignant and invasive phenotype of cutaneous SCC. Functional evidence for detrimental effects of loss of T-cadherin expression on SCC behaviour was provided through gain- and loss-of-function studies using the SCC cell line A413. In 2D culture models, silencing of $\mathrm{T}$-cadherin gene expression increased proliferation, random motility and transmigration across a matrix barrier [20]. In a 3D culture model (multicellular spheroids embedded in collagen I or Matrigel matrices), silencing of T-cadherin markedly increased outward expansion/invasion into the matrices [20]. Further, use of the murine xenograft model showed that whereas xenografts composed of SCC expressing T-cadherin exhibited a closed invasion front pattern, xenografts composed of T-cadherin-silenced SCC were poorly circumscribed, with an invasion front characterized by an irregular interface at the advancing edge of the tumour and formation of small irregularly shaped nests and cords of cells dissecting through dermal collagen [23]. Moreover, in the xenograft model, loss of T-cadherin resulted in increased metastatic potential and aggressiveness of SCC [24].

The pathogenesis of AK progression into iSCC is currently based on two main pathways, namely classical and differentiated. In the classical pathway, acquisition of dermal invasiveness occurs only after complete epidermal hyperplasia through stepwise progression from AK I to AK II and AK III. In the differentiated pathway, iSCCs directly arise from atypical keratinocytes that are present only in the basal layer (AK I) $[2,8]$. A recent search for molecular markers that distinguish the two pathways found that iSCCs arising through the classical pathway more frequently exhibited higher expression of the proliferation marker Ki67, whereas iSCCs arising through the differentiated pathway more frequently exhibited activation of the epithelial-tomesenchymal transition programme (characterized by acquisition of vimentin expression and loss of E-cadherin and $\beta$-catenin) [25]. We suggest that T-cadherin may represent another marker of pathway distinction since T-cadherin-negative iSSCs most commonly associated with type AK I (i.e., following the differentiated pathway), whereas T-cadherin-positive iSCCs most commonly originated from AK II and AK III (i.e., following the classical pathway).

Since without treatment some AK lesions may undergo malignant transformation into iSCC [26], current recommendations for clinical practice suggest that all AK lesions along with the actinically damaged field (field cancerization) $[8,27]$ should be monitored and adequately treated in order to prevent possible invasion, metastasis and even mortality [7, 28, 29]. Clinical changes in a lesion, such as induration, inflammation, rapid growth, bleeding and ulceration, may indicate progression to a more advanced stage [30,31]. Definitive distinction of an AK from early in situ iSCC can only be made by histological means, and prediction of outcome of AK based on clinical and histological features alone is difficult. A limitation of the present immunohistochemical study is the small number of specimens examined. Nevertheless, based on our present findings and previous investigations [20,23, 24] we speculate that $\mathrm{T}$-cadherin may represent a biomarker for predicting AK outcome: loss of T-cadherin in the basal keratinocyte layer of AK regardless of the downward or upward patterns of proliferation may be indicative of potential malignant transformation, whereas the persistent presence of T-cadherin may indicate a more benign course of disease progression, or even a spontaneous regression of the lesion. 


\section{Statement of Ethics}

The authors have no ethical conflicts to disclose.

\section{Disclosure Statement}

The authors have no conflicts of interest to declare.

\section{Author Contributions}

S.A.B. and T.J.R. conducted histological and immunohistochemical analyses, and wrote the manuscript.

\section{References}

1 Ratushny V, Gober MD, Hick R, Ridky TW, Seykora JT. From keratinocyte to cancer: the pathogenesis and modeling of cutaneous squamous cell carcinoma. J Clin Invest. 2012 Feb;122(2):464-72.

2 Fernández-Figueras MT, Carrato C, Sáenz X, Puig L, Musulen E, Ferrándiz C, et al. Actinic keratosis with atypical basal cells (AK I) is the most common lesion associated with invasive squamous cell carcinoma of the skin. J Eur Acad Dermatol Venereol. 2015 May;29(5):991-7.

3 Green AC, Olsen CM. Cutaneous squamous cell carcinoma: an epidemiological review. Br J Dermatol. 2017 Aug; 177(2):373-81.

4 Czarnecki D, Meehan CJ, Bruce F, Culjak G. The majority of cutaneous squamous cell carcinomas arise in actinic keratoses. J Cutan Med Surg. 2002 May-Jun;6(3):207-9.

5 Mittelbronn MA, Mullins DL, Ramos-Caro FA, Flowers FP. Frequency of pre-existing actinic keratosis in cutaneous squamous cell carcinoma. Int J Dermatol. 1998 Sep;37(9):677-81.

6 Röwert-Huber J, Patel MJ, Forschner T, Ulrich C, Eberle J, Kerl H, et al. Actinic keratosis is an early in situ squamous cell carcinoma: a proposal for reclassification. Br J Dermatol. 2007 May;156(s3 Suppl 3):8-12.

7 Werner RN, Stockfleth E, Connolly SM, Correia O, Erdmann R, Foley P, et al.; International League of Dermatological Societies; European Dermatology Forum. Evidence- and consensus-based (S3) Guidelines for the Treatment of Actinic Keratosis - International League of Dermatological Societies in cooperation with the European Dermatology Forum - Short version. J Eur Acad Dermatol Venereol. 2015 Nov;29(11):2069-79.

8 Fernandez Figueras MT. From actinic keratosis to squamous cell carcinoma: pathophysiology revisited. J Eur Acad Dermatol Venereol. 2017 Mar;31 Suppl 2:5-7.

9 Schmitz L, Gambichler T, Kost C, Gupta G, Stücker M, Stockfleth E, et al. Cutaneous squamous cell carcinomas are associated with basal proliferating actinic keratoses. Br J Dermatol. 2018 Mar. https://doi.org/10.1111/ bjd.16536.

10 Heerfordt IM, Nissen CV, Poulsen T, Philipsen PA, Wulf HC. Thickness of Actinic Keratosis Does Not Predict Dysplasia Severity or P53 Expression. Sci Rep. 2016 Sep;6(1):33952.

11 Schmitz L, Kahl P, Majores M, Bierhoff E, Stockfleth E, Dirschka T. Actinic keratosis: correlation between clinical and histological classification systems. J Eur Acad Dermatol Venereol. 2016 Aug;30(8):1303-7.

12 Schmitz L, Gambichler T, Gupta G, Stücker M, Stockfleth E, Szeimies RM, et al. Actinic keratoses show variable histological basal growth patterns - a proposed classification adjustment. J Eur Acad Dermatol Venereol. 2018 May;32(5):745-51.

13 Angst BD, Marcozzi C, Magee AI. The cadherin superfamily: diversity in form and function. J Cell Sci. 2001 Feb; 114(Pt 4):629-41.

14 Tinkle CL, Pasolli HA, Stokes N, Fuchs E. New insights into cadherin function in epidermal sheet formation and maintenance of tissue integrity. Proc Natl Acad Sci USA. 2008 Oct;105(40):15405-10.

15 Saito M, Tucker DK, Kohlhorst D, Niessen CM, Kowalczyk AP. Classical and desmosomal cadherins at a glance. J Cell Sci. 2012 Jun;125(Pt 11):2547-52.

16 Philippova M, Joshi MB, Kyriakakis E, Pfaff D, Erne P, Resink TJ. A guide and guard: the many faces of T-cadherin. Cell Signal. 2009 Jul;21(7):1035-44.

17 Zhou S, Matsuyoshi N, Liang SB, Takeuchi T, Ohtsuki Y, Miyachi Y. Expression of T-cadherin in Basal keratinocytes of skin. J Invest Dermatol. 2002 Jun;118(6):1080-4.

18 Buechner SA, Philippova M, Erne P, Mathys T, Resink TJ. High T-cadherin expression is a feature of basal cell carcinoma. Br J Dermatol. 2009 Jul;161(1):199-202. 
19 Buechner S, Erne P, Resink TJ. T-Cadherin Expression in the Epidermis and Adnexal Structures of Normal Skin. Dermatopathology (Basel). 2016 0ct;3(4):68-78.

20 Pfaff D, Philippova M, Buechner SA, Maslova K, Mathys T, Erne P, et al. T-cadherin loss induces an invasive phenotype in human keratinocytes and squamous cell carcinoma (SCC) cells in vitro and is associated with malignant transformation of cutaneous SCC in vivo. Br J Dermatol. 2010 Aug;163(2):353-63.

21 Takeuchi T, Liang SB, Matsuyoshi N, Zhou S, Miyachi Y, Sonobe H, et al. Loss of T-cadherin (CDH13, H-cadherin) expression in cutaneous squamous cell carcinoma. Lab Invest. 2002 Aug;82(8):1023-9.

22 Cassarino DS, Derienzo DP, Barr RJ. Cutaneous squamous cell carcinoma: a comprehensive clinicopathologic classification-part two. J Cutan Pathol. 2006 Apr;33(4):261-79.

23 Pfaff D, Philippova M, Kyriakakis E, Maslova K, Rupp K, Buechner SA, et al. Paradoxical effects of T-cadherin on squamous cell carcinoma: up- and down-regulation increase xenograft growth by distinct mechanisms. J Pathol. 2011 Dec;225(4):512-24.

24 Philippova M, Pfaff D, Kyriakakis E, Buechner SA, Iezzi G, Spagnoli GC, et al. T-cadherin loss promotes experimental metastasis of squamous cell carcinoma. Eur J Cancer. 2013 May;49(8):2048-58.

25 Saenz-Sardà X, Carrato C, Pérez-Roca L, Puig L, Ferrándiz C, Ariza A, et al. Epithelial-to-mesenchymal transition contributes to invasion in squamous cell carcinomas originated from actinic keratosis through the differentiated pathway, whereas proliferation plays a more significant role in the classical pathway. J Eur Acad Dermatol Venereol. 2018 Apr;32(4):581-6.

26 Marks R, Rennie G, Selwood TS. Malignant transformation of solar keratoses to squamous cell carcinoma. Lancet. 1988 Apr;1(8589):795-7.

27 Stockfleth E. The importance of treating the field in actinic keratosis. J Eur Acad Dermatol Venereol. 2017 Mar; 31 Suppl 2:8-11.

28 Hofbauer G, Anliker M, Boehncke WH, Brand C, Braun R, Gaide O, et al. Swiss clinical practice guidelines on field cancerization of the skin. Swiss Med Wkly. 2014 Dec;144:w14026.

29 de Oliveira EC, da Motta VR, Pantoja PC, Ilha CS, Magalhães RF, Galadari H, et al. Actinic keratosis - review for clinical practice. Int J Dermatol. 2018 Aug. https://doi.org/10.1111/ijd.14147.

30 Quaedvlieg PJ, Tirsi E, Thissen MR, Krekels GA. Actinic keratosis: how to differentiate the good from the bad ones? Eur J Dermatol. 2006 Jul-Aug;16(4):335-9.

31 Rosen T, Lebwohl MG. Prevalence and awareness of actinic keratosis: barriers and opportunities. J Am Acad Dermatol. 2013 Jan;68(1 Suppl 1):S2-9. 